\title{
Cell Death Delineates Axon Pathways in the Hindlimb and Does So Independently of Neurite Outgrowth
}

\author{
Kathryn W. Tosney, ${ }^{1}$ Sally Schroeter, and Jennifer A. Pokrzywinski \\ Department of Biology, Natural Science Building, The University of Michigan, Ann Arbor, Michigan 48109
}

Accepted August 17, 1988

\begin{abstract}
We wished to know whether the cell death and phagocytosis seen near the outgrowing nerve front in the hindlimb delineate axon pathways and, if so, whether the cells died only in the presence of growth cones. We unilaterally deleted the lumbosacral neural tube and reconstructed the patterns of neurite outgrowth and phagocytes during the stage when neurites first begin to colonize the thigh. In the control limbs, sensory and motor nerve pathways coincided with sites of phagocytosis, including those pathways that had yet to be colonized by growth cones. For instance, phagocytes were clustered at foci within the muscle masses where muscle nerves form a day later. However, they were not seen in adjacent, nonpathway regions such as posterior sclerotome or dorsal and ventral to the region of the plexus in which axons extend only posteriorly. Phagocytes were also seen in defined regions that are probably inaccessible to growth cones because they are too distant from pathways (i.e., subjacent to the apical ectodermal ridge) or express substances that are typical of precartilagenous tissues which may prohibit axon advance. In the experimental limbs, we conservatively estimated that neurite outgrowth was reduced to less than one-tenth (neurites were visible only with electron microscopy) or less than one-third of normal. Outgrowth extended less far distally and, in half the cases, motor innervation was completely abolished. Despite the extensive reduction in neurite outgrowth, the distribution of phagocytes was indistinguishable from that of the control side. Furthermore, the number of phagocytes did not differ significantly. We conclude that cell death delineates axon pathways remarkably well and does so without an interaction with growth cones; it is an independent characteristic of the axonal pathways and may be directly or indirectly important to axonal pathfinding. This is the first identification of a feature that characterizes prospective nerve pathways in the hindlimb. (c) 1988 Academic Press, Inc.
\end{abstract}

\section{INTRODUCTION}

Motoneurons that enter the chick limb follow stereotyped pathways that appear to be delineated by local features of the environment (Swanson and Lewis, 1982; Tosney and Landmesser, 1984; Stirling and Summerbell, 1985; see review by Landmesser, 1984). Despite strong evidence that these pathways are more permissive for outgrowth than adjacent regions, no molecular or cellular feature has been found that characterizes the pathways before growth cones colonize them, with one possible exception: cells die and are phagocytized in the vicinity of growth cones as they enter the major nerve trunk pathways in the hindlimb (Tosney and Landmesser, 1985a). We extended this earlier study by mapping, in detail, the distribution of phagocytes within the hindlimb during the stage when the first growth cones normally colonize the thigh. We report here that the spatial pattern of axon pathways coincides with regions where dead cells have been phagocytized. This correlation is consistent with the intriguing

\footnotetext{
${ }^{1}$ To whom correspondence should be addressed.
}

possibility that the death of cells in some way helps to establish the pathways.

It is possible that the death of these cells could result from an interaction with growth cones. This view is consistent with the pattern of cell death and some known properties of growth cones. The greatest density of dying cells has been suggested to coincide with the distribution of growth cones; in adjacent nonpathway regions and in the more proximal nerve where growth cones are absent, dead cells and phagocytes were seldom seen (Tosney and Landmesser, 1985a). In addition, growth cones release a number of molecules, including plasminogen activator (cf. Kryostosek and Seeds, 1981; Patterson, 1985; Pittman, 1985), that could alter the micromilieu of cells and lead to their demise.

We essentially asked whether this cell death was "murder" (required an interaction with growth cones) or "suicide" (independent of neural interactions and thus directly or indirectly characteristic of whatever processes generate the pathways). We diminished neurite outgrowth to less than one third of normal and, in half the cases, completely prevented the outgrowth of motor axons. We then determined whether cells still died, did so predominantly within pathways, and in 
their normal abundance. We found that they did all of the above, suggesting that cell death is an overt characteristic of the emerging axonal pathways in the limb.

A brief report of this work has appeared elsewhere (Schroeter et al., 1988).

\section{MATERIALS AND METHODS}

Embryonic surgery. Stage 17 and 18 (Hamburger and Hamilton, 1951) white leghorn chick embryos (Michigan State University) were lightly stained with $0.25 \%$ neutral red in sterile water. We used a modification of the ablation method described by Landmesser and Honig (1986). The neural tube was split open with a tungsten needle at the lumbosacral level and the neural tube was removed unilaterally using aspiration through a small micropipet (tip size, $20-40 \mu \mathrm{m}$ ). The embryo was moistened with sterile saline containing 100 units $/ \mathrm{ml}$ of penicillin-streptomycin (GIBCO) and incubated until stage 25.

We chose to operate at stages 17 and 18, just before and during neural crest emigration, since we hoped to produce two classes of embryos: (1) those without either sensory or motor neurites, and (2) those in which dorsal root ganglia (DRG) had formed, but motor innervation was absent. If neurites had an effect on the pattern or abundance of cell death in the limb, we could compare these two classes to detect whether only the motor or only the sensory population was essential to cell death. In addition, these embryos are more robust than younger embryos and develop fewer abnormalities following surgery.

Histology and electron microscopy. We used a method, described in detail elsewhere (Tosney and Landmesser, 1986), in which neurites and phagocytes can be observed in serial thick sections that are suitable for later analysis with transmission electron microscopy (EM). Briefly, at stage 25 (when growth cones are normally invading the thigh), operated embryos were decapitated, eviscerated, and fixed in $2.5 \%$ glutaraldehyde in $0.1 M$ cacodylate buffer for $18-24 \mathrm{hr}$. They were postfixed in $2 \%$ osmium tetroxide in $0.1 M$ cacodylate buffer overnight at $4^{\circ} \mathrm{C}$, dehydrated in graded alcohols, and embedded in Epon-Araldite through propylene oxide. The polymerized block was trimmed and the embryos were serially sectioned at $25 \mu \mathrm{m}$ with a heated steel knife on a standard rotary microtome. Thin sections were obtained from selected thick sections, mounted on formvar-coated single hole grids, stained with uranyl acetate and lead citrate, and viewed in a Philips 300 electron microscope. We preferred this over histological methods that have been used to detect cell death, such as staining with neutral red or nile blue (see Saunders and Fallon, 1967), because these dyes often fail to penetrate the deeper regions of the limb where the major axonal pathways lie. In addition, our method gave us the option of confirming details at the ultrastructural level.

Distribution and abundance of phagocytes. Regions of cell death typically have an abundance of phagocytes that are easily identifiable in plastic sections (see Tosney and Landmesser, 1985a); these cells have been used as the primary indicator of cell death in other regions of the limb (cf. Saunders and Fallon, 1967). We have also used the presence of phagocytes to indicate regions of cell death.

We chose for detailed analysis six embryos that fit the following criteria. (1) Both limbs were normal in morphology and similar in size. (2) Neurite outgrowth into the control limb was normal in origin, distal extent, and abundance. (3) Neurite outgrowth was severely reduced on the experimental side. This gave us an internal control for comparing the numbers and patterns of phagocytes and avoided possible variation due to differences in processing.

We recorded the distribution of the phagocytes on camera lucida tracings of every other $25-\mu \mathrm{m}$ section through the lumbosacral region. We also recorded the positions of the lateral motor columns, DRG, neurite outgrowth, myotomes, major blood vessels, muscle masses, and cartilage primordia. We reconstructed the limbs from a dorsal view to assess the abundance and extent of axon outgrowth and the spatial relation between the pathways and distribution of phagocytes along the anterior-posterior and medial-lateral axes. We confirmed the magnitude and source of nerve outgrowth and the distribution of phagocytes in selected thick sections with EM. Several thin sections from each thick section were examined in order to detect all the phagocytes (which were generally smaller in diameter than the thick section) and small axon fascicles.

We classified sets of pathways into two major classes: the crural region in the anterior thigh, served by lumbosacral segments $1-3$, and the sciatic region in the posterior thigh, served by lumbosacral segments 3-8. Each region contains a plexus region and pathways for spinal nerves, dorsal and ventral nerve trunks, and associated cutaneous and muscle nerves. Of the twelve pathway regions examined on the experimental sides (crural and sciatic regions of six embryos), motor axons were absent in six, sensory neurites were absent in one, and the remainder had small mixed nerves. We counted the number of phagocytes in camera lucida drawings of every other section on control and experimental sides to estimate the magnitude of the cell death in each region and in each type of pathway within each region. The 
number on the control side did not differ significantly from that found in unoperated embryos (not shown). Significant differences between means were determined using Student's $t$ test.

\section{RESULTS}

Anatomy of operated embryos and innervation of control limbs. The control and experimental limbs of the embryos selected for analysis were all normal in size and morphology, as illustrated in Figs. 1A and 1B. In each limb the dorsal and ventral muscle masses were distinct but had not yet separated to form individual muscles and the primordia of the pelvic girdle and femur-tibiotarsus were clearly distinguishable. These structures merged gradually with a more distal homogeneous mesenchyme that extended up to the apical ectodermal ridge. Since the spinal cord was absent on the experimental sides, the remaining portion of the cord generally lay open. Nevertheless, the DRG and lateral motor columns were normal in size in each segment of the control sides.

The normal nerve pattern was displayed in all control limbs and the nerves were normal in size and distal extent (see control side of Fig. 5). The limb is served by motor and sensory neurites from the eight lumbosacral segments. The nerve patterns in the limb can be conveniently classified into two similar but distinct regions, the anterior crural region served by segments 1-3 and the posterior sciatic region served by segments 3-8. These regions were analyzed separately. In each region the sets of pathways are similar; axons that have tra- versed the spinal nerve pathways in the anterior sclerotome and converged within the crural or sciatic plexus region have begun to colonize the respective dorsal and ventral nerve trunk pathways in each region at stage 25 , the stage we examined. The muscle nerves will branch from the nerve trunk pathways a day later, at stage 26.5.

Identification of phagocytes with light and electron $m i$ croscopy. We used the distribution of cells with a distinct appearance in bright-field or Nomarski optics as indicators of cell death. These cells are round, are relatively translucent, contain a number of dark granules, and are clearly distinct from irregularly shaped mesenchyme cells (Fig. 2A). When we ultrastructurally examined regions where we had seen these cells at the light microscopic level, we could readily identify the same cells. As Fig. 2B shows, they are phagocytes filled with cellular debris. The phagocytes do not resemble frank macrophages and are likely to be mesenchyme cells that have engulfed their dying neighbors. The phagocytes in other necrotic limb regions have been proposed to originate from surrounding mesenchyme cells in this cannibalistic manner and the large amount of debris contained within such cells suggests that cell death is quite abundant (see Saunders and Fallon, 1967).

We were initially concerned that we could not readily identify all the phagocytes at the light microscopic level. However, when we carefully noted the position of phagocytes with camera lucida tracings or photographs and then examined the same regions with electron microscopy, we did not find additional phagocytes. For example, Figs. $3 \mathrm{~A}$ and $3 \mathrm{~B}$ show light and electron mi-
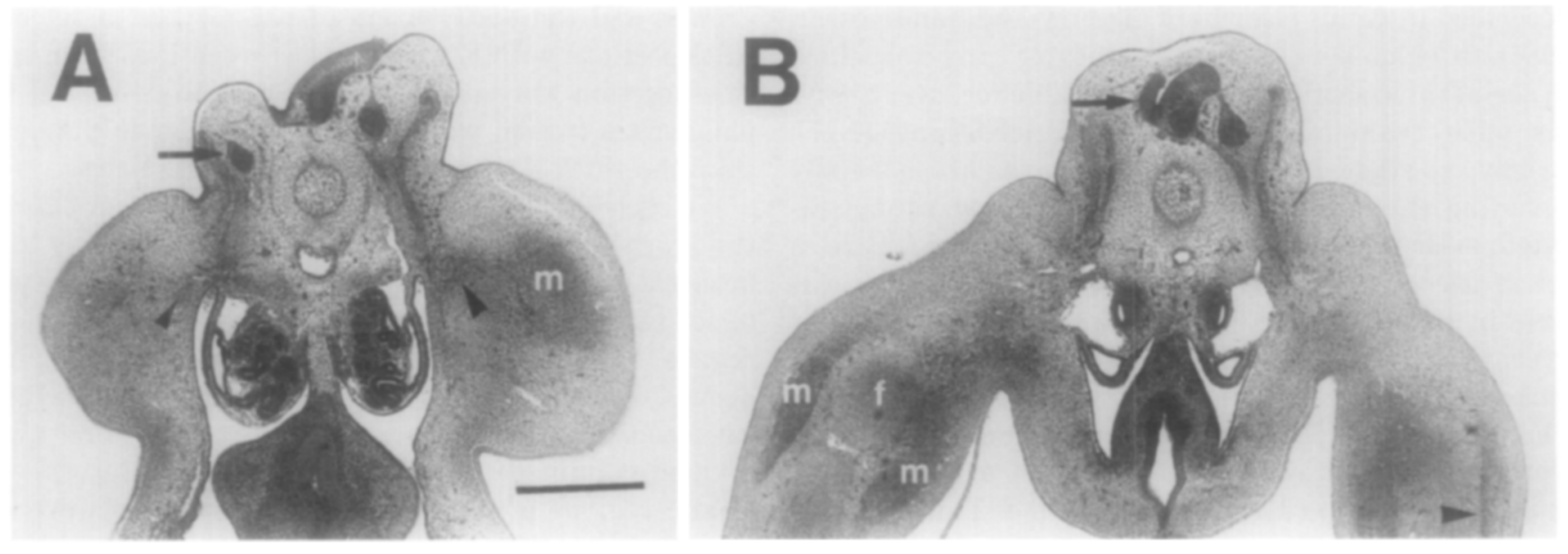

FIG. 1. The operated side is to the left in each of these cross sections. (A) A small DRG (arrow) has formed but the motoneurons have been completely deleted on the operated side in the crural region of this embryo. The operated limb looks smaller because the section is somewhat oblique. Figure 7 shows electron micrographs from the regions indicated by arrowheads. (B) The DRG has not formed in this segment of the sciatic region but a portion of the ventral spinal cord (arrow) remains on the operated side. The region indicated by an arrowhead is shown at higher magnification in Fig. 3. m, muscle mass; f, femur-tibiotarsus primordium. A reconstruction of this embryo is shown in Fig. 5 . Calibration bar $=500 \mu \mathrm{m}$. 


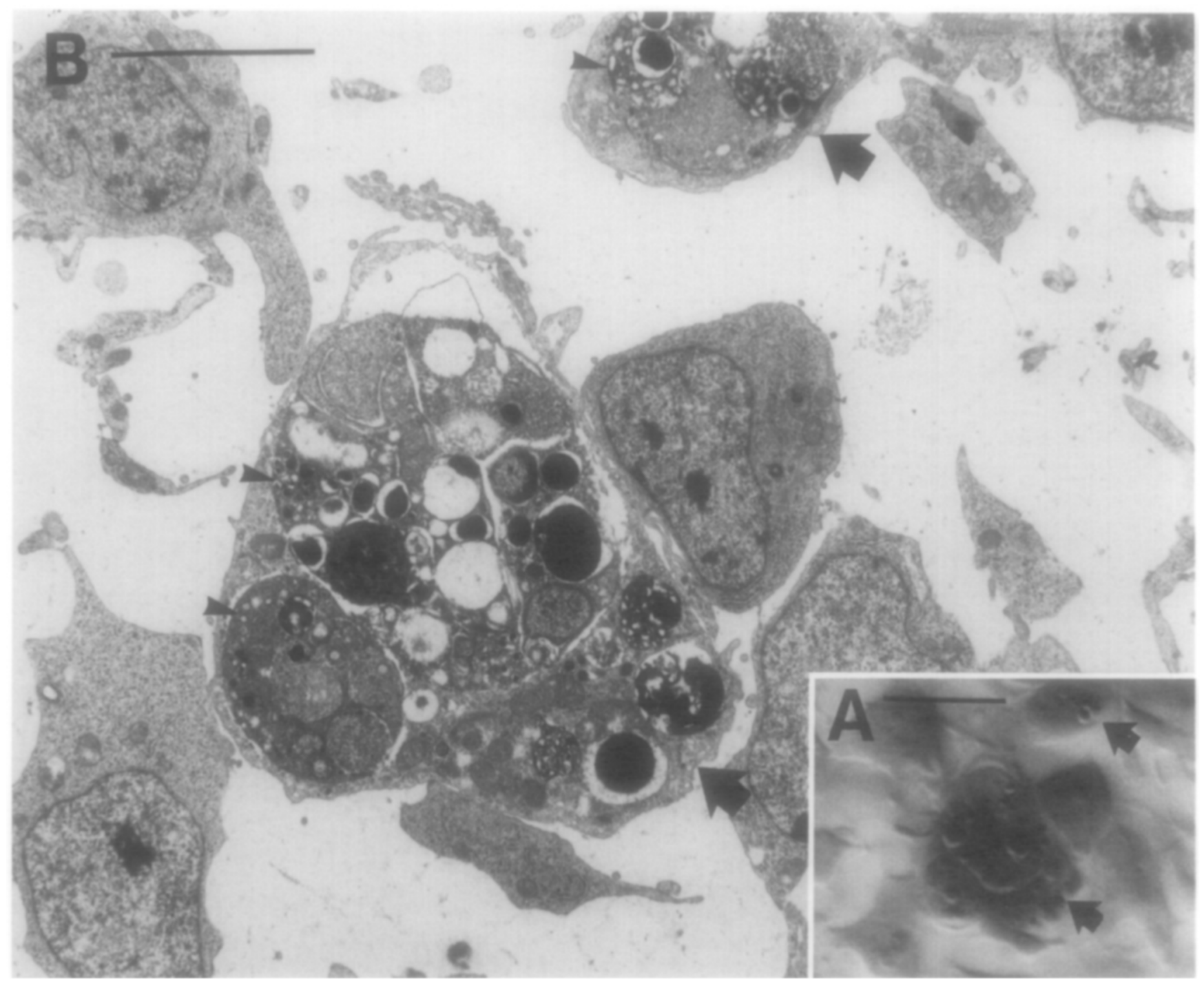

FIG. 2. Identification of phagocytes with light and electron microscopy. (A) Two phagocytes (arrows) viewed with Nomarski optics are typically large, rounded, and filled with numerous dark granules. (B) The same cells (arrows) can be located in thin sections and typically contain large amounts of engulfed cellular debris (arrowheads). Calibration bars $=10 \mu \mathrm{m}$.

crographs of the same area. Only a single phagocyte is detectable with either method. We therefore feel confident that we could clearly distinguish phagocytes in thick sections and that they were absent in regions where we did not detect them at the light microscopic level.

Spatial pattern of cell death in control limbs. We consistently saw phagocytes in three regions that growth cones do not colonize. (1) Phagocytes lay within the loose mesenchyme subjacent to the anterior portion of the apical ectodermal ridge (Fig. 4A and ANZ in Figs. 5 and $6 \mathrm{~A}$ ), a region previously described as the "anterior necrotic zone" (cf. Saunders et al., 1962). (2) Scattered phagocytes were seen within the developing head of the femur and were abundant at the femur-tibiotarsus junction (Fig. 4B and "op" in Figs. 6B and 6C). The latter necrotic region has been called the "opaque patch" (cf. Dawd and Hinchliffe, 1971). (3) Numerous phagocytes were present in the loose mesenchyme along the outer edge of the myotome (see Figs. 6A-6D), as previously described by Jacob et al. (1979; see also Tosney and Landmesser, 1985a). Reasons to believe that these regions are not accessible for growth cone ingress are addressed in the discussion. While the phagocytes in these regions had the typical appearance described above for those that lie along nerve pathways, many appeared to be larger and to enclose more cellular debris (Fig. 4C). 


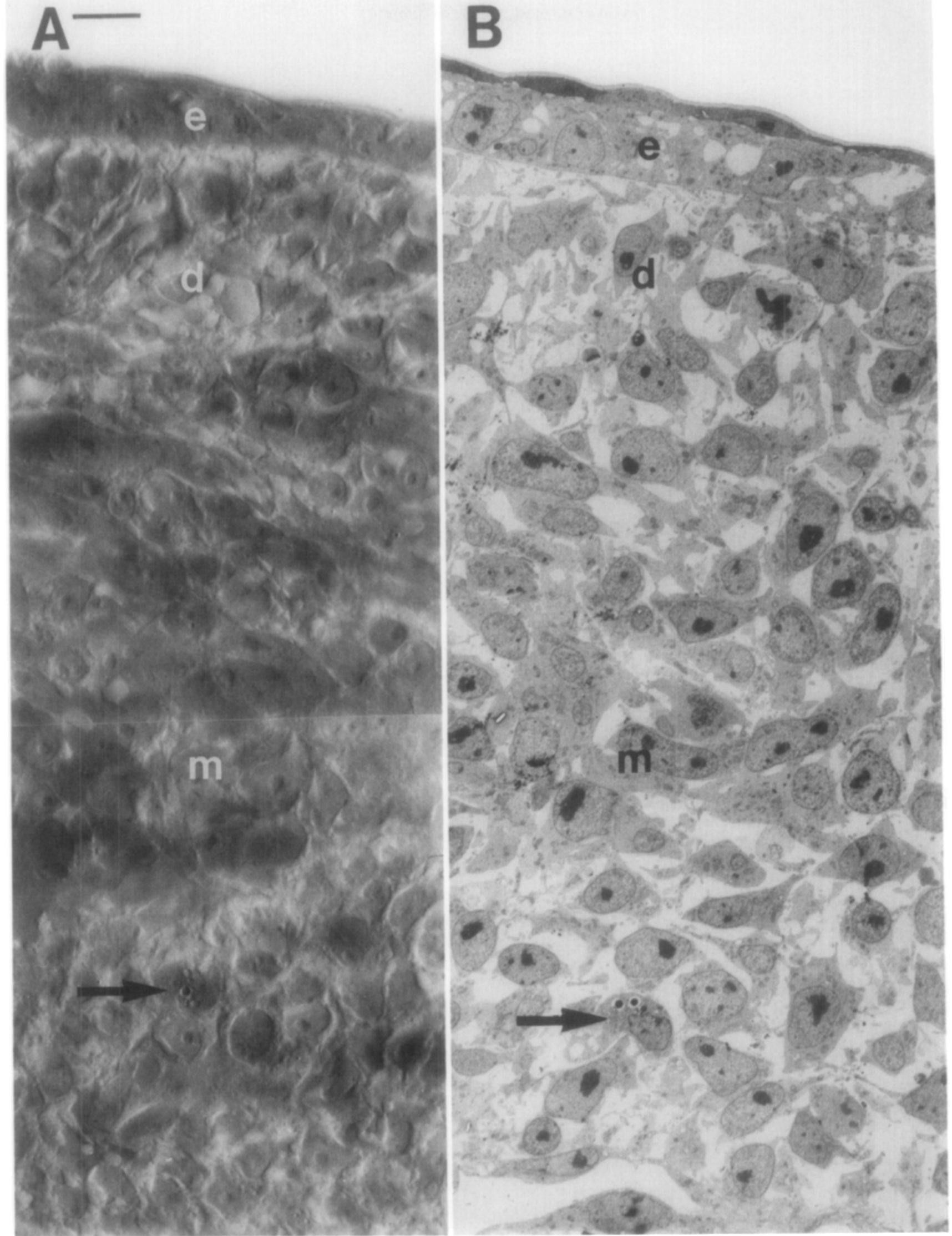


The control side of Fig. 5 shows a representative reconstruction of the normal distribution of phagocytes relative to nerve outgrowth as viewed from the dorsal surface. This view enhances visualization of patterns along the anterior-posterior and medial-lateral axes. This reconstruction clearly shows that phagocytes are distributed in a spatial pattern that coincides with pathways. In fact, phagocytes are scattered distally beyond the growing nerve front into the pathways that growth cones will later traverse (e.g., in Fig. 5, Acut, anterior cutaneous nerve trunk path; $\mathrm{C}$, crural nerve trunk path; S, sciatic nerve trunk path; ax, axial nerve path; and obt, the obturator or ventral crural nerve trunk path). Phagocytes along nerve trunk pathways were up to $400 \mu \mathrm{m}$ from the most distal tip of the outgrowing nerve. Cell death, therefore, appears to presage neurite advance. In addition, it appears to be temporally correlated with growth cone advance since phagocytes are absent in the most proximal pathway regions (i.e., the proximal portion of the spinal nerve pathways, sn in Fig. 5) which the majority of the growth cones have already traversed.

We were particularly struck by the absence of phagocytes in areas where growth cones do not ramify. For instance, we never saw phagocytes in the posterior sclerotome but always found a few in the anterior sclerotome, along the spinal nerve pathway (sn in Fig. 5 ). In addition, phagocytes were confined to the corresponding crural or sciatic nerve trunk pathways within the limb except at one point, where they bridge dorsally over the central cartilagenous tissue. This is the sole region where a muscle, the iliotibialis, normally receives a dorsal nerve branch from both crural and sciatic nerve trunks (itib in Fig. 5). Phagocytes were also prominent in the loose mesenchyme that the anterior cutaneous nerve (Acut in Fig. 5) will later traverse, but were rarely seen in similar mesenchyme elsewhere.

The remarkable correspondence between the distribution of phagocytes and individual nerve pathways is more easily visualized in camera lucida tracings that preserve the dorsoventral relationships in the limb. The typical pattern is illustrated on the control sides of Figs. $6 \mathrm{~A}-6 \mathrm{D}$; the abbreviations in parentheses below refer to these figures.

In the crural region illustrated in Fig. $6 \mathrm{~A}$, phagocytes are prominant along the spinal nerve $(\mathrm{sn})$, within the plexus region, along the ventral (obt) nerve trunk path, and along the portion of the dorsal (De) nerve trunk path that will branch and end within the femorotibialis muscle. They are present along the anterior (Acut) and posterior (Pcut) cutaneous pathways as well as along pathways that contain both motor and sensory nerves. In each case, they also delineate distal portions of the pathways that growth cones have yet to enter. In particular, they are present at foci within the dorsal muscle mass itself. These foci (dd, itib, and others described below) appear to correspond to regions where particular muscle nerves will subsequently form. Although we do not have an independent marker for muscle nerve sites at this early stage, these nerves will form in stereotyped positions. As far as we can determine, the foci of phagocytes within muscle masses correspond remarkably well to the positions where nerves would be expected to invade the muscle masses. In addition, at this stage we detected foci corresponding to every one of the muscle nerves that will form in the thigh.

The distribution of phagocytes in the more posterior crural region which lies between crural and sciatic nerve trunk pathways is illustrated in Fig. 6B. Foci of phagocytes are present in the dorsal muscle mass. The most proximal corresponds to the path to the intrinsic, "deep dorsal" (dd) muscles of the dorsal girdle; another marks the path of the anterior iliotibialis (itib) muscle nerve; the most distal marks the continuing path of the dorsal crural (Dc) nerve trunk. Ventral to the femur (F), foci of phagocytosis correspond to the adductor (add) muscle nerve pathway and to a continuation of the ventral nerve trunk pathway. One striking difference is seen in the plexus region, where there are few phagocytes and these are confined to a very small area. This is in dramatic contrast to the wide distribution of phagocytes in the plexus regions that lie immediately toward the anterior and posterior (compare to Figs. 6A and $6 \mathrm{C}$ ). This local pattern corresponds precisely with the local nerve pathway: neurites in this region of the plexus are within the ramus communicans (rc) pathway that conveys neurites from segments 3 and 4 into the sciatic plexus region where they join neurites from segments 5 to 8 . These axons travel only in a posterior direction and they do not ramify in dorsal or ventral directions where, intriguingly, there are no phagocytes. The sciatic region is illustrated in Fig. 6C. Here, the

FIG. 3. Identification and distribution of phagocytes. (A) A single phagocyte (arrow) is identifiable in this $25-\mu \mathrm{m}$ plastic section viewed with Nomarski optics. (B) The same phagocyte (arrow) can also be identified in an electron micrograph of the same region. This phagocyte is within the mesenchyme along the distal portion of the dorsal sciatic nerve trunk pathway (see arrow in Fig. 1B). Additional phagocytes can not be discerned in any of the semiserial thin sections taken from this thick section. This suggests that these cells could readily be identified using light microscopic criteria. Ultrastructural surveys of nonpathway regions like the muscle mass $(\mathrm{m})$ and dermis $(\mathrm{d})$ in this region did not reveal phagocytes that we had missed at the light microscopic level. e, ectoderm. Calibration bar $=10 \mu \mathrm{m}$. 

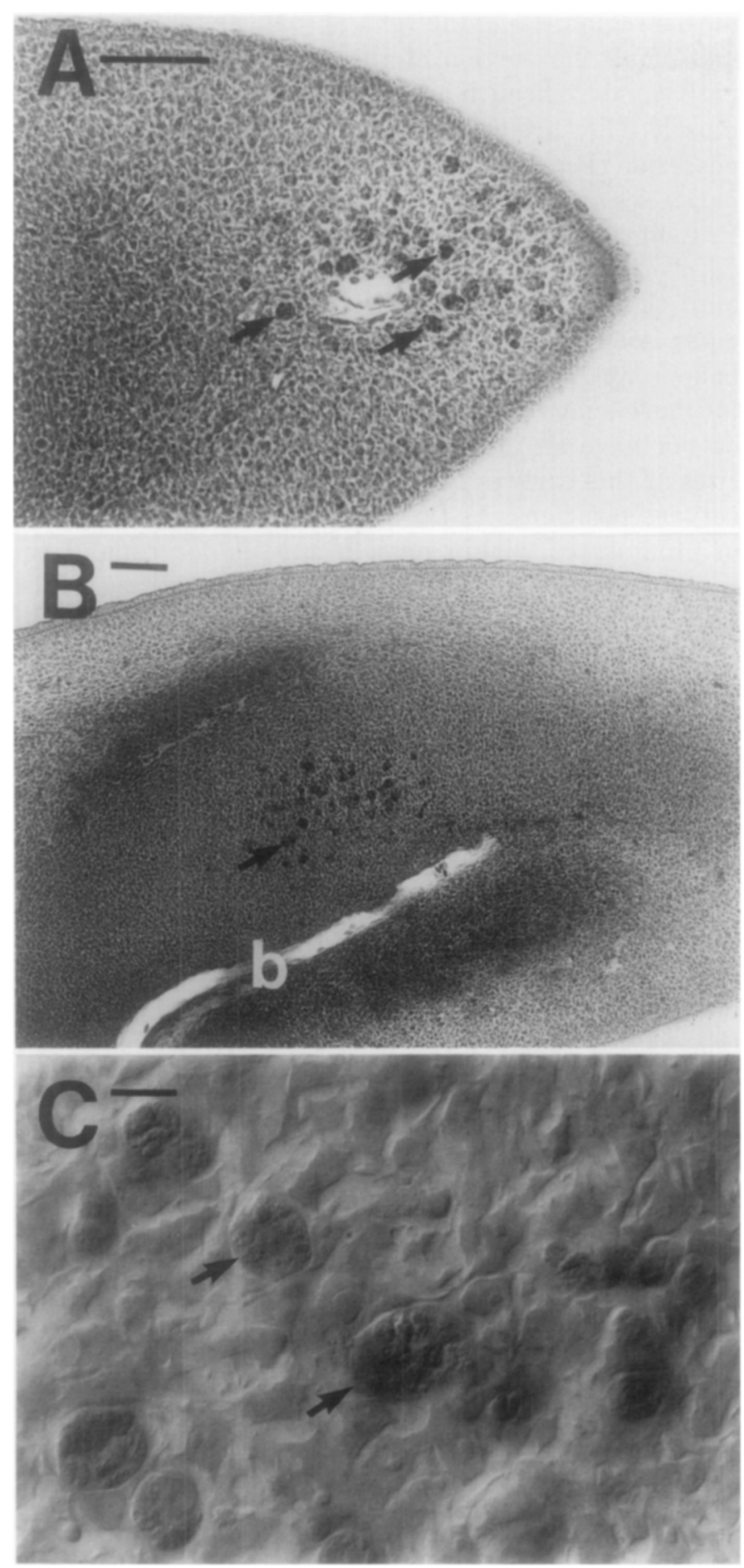

FIG. 4. Phagocytes (arrows) were typically found in two regions of the limb that are not axonal pathways: $(A)$ in the "anterior necrotic zone" subjacent to the apical ectodermal ridge, and (B) in the "opaque patch" within the femur-tibiotarsus condensation. (C) Phagocytes in these regions viewed at higher magnification are enlarged, are rounded, contain granules, and are similar to the phagocytes seen along axonal pathways. $\mathrm{b}$, blood vessel. Calibration bars $=10 \mu \mathrm{m}$.

plexus region is particularly spacious; axons ramify over a broader region than elsewhere in the leg and phagocytes are scattered over a correspondingly larger region. Phagocytes also delineate the dorsal (Ds) and ventral (Vs) sciatic nerve trunk pathways and are present at foci within the dorsal muscle masses that correspond to sites of muscle nerve formation (dd, ifib, itib). They are particularly abundant along the paths where muscle nerves will diverge from the ventral nerve trunk into the axial (ax) muscles in the lateral body wall and the deep ventral (dv), ischioflexorius (ischio), and caudilioflexorius (caud) muscles of the limb.

In the sciatic region posterior to the major nerve trunks (Fig. 6D), the distribution of phagocytes is much more restricted than it is to the anterior. These cells are seen along the spinal nerve (sn) and axial (ax) pathways and in the plexus region where axons from LS7 and LS8 are joining. The few phagocytes shown within the limb were present only in the more anterior sections and may be distal continuations of pathways that began more anteriorly. They are absent elsewhere.

We also looked for correlations between the above spatial pattern of phagocytes and limb features other than axonal pathways. In particular, we asked whether this phagocytosis also correlated with sites where tissue masses were separating. Phagocytes were found along some planes where tissue masses had recently become distinct and did not extend distally into regions where tissue borders were still undelineated. For instance, they were seen between muscle masses and the femur-tibiotarsus condensation, within the plane where the femur and tibiotarsus will separate, surrounding portions of the developing pelvic girdle, and between the myotome and dermal tissues. However, nerve trunk pathways largely lie within planes between tissues at this stage and phagocytes were not found in all regions of tissue separation. In particular, phagocytes were generally absent or sparse in planes that are adjacent to but do not provide axonal pathways such as around the surface of the pelvic girdle that faces the limb, along the inner surface of the pelvic girdle in the posterior, dorsal and ventral to the ramus communicans pathway, and posteriorly between the muscle masses and the chondrogenic core.

We also addressed the spatial relationship among phagocytes, axon pathways, and blood vessels. When we examined sections with EM, we very rarely saw vascular endothelium surrounding a phagocyte. The phagocytes are not, therefore, traveling within the vasculature and bound for distant destinations. In addition, we confirmed that the major vessels, while occasionally paralleling axon pathways, do not delineate them (cf. Tosney and Landmesser, 1985a). We did not chart the distribution of fine capillaries and it remains possible that axons preferentially colonize areas of rich vascularization. Alternatively, since the limb vasculature is undergoing extensive remodeling during this period (cf. Caplan, 1985), it is possible that axons enter regions 


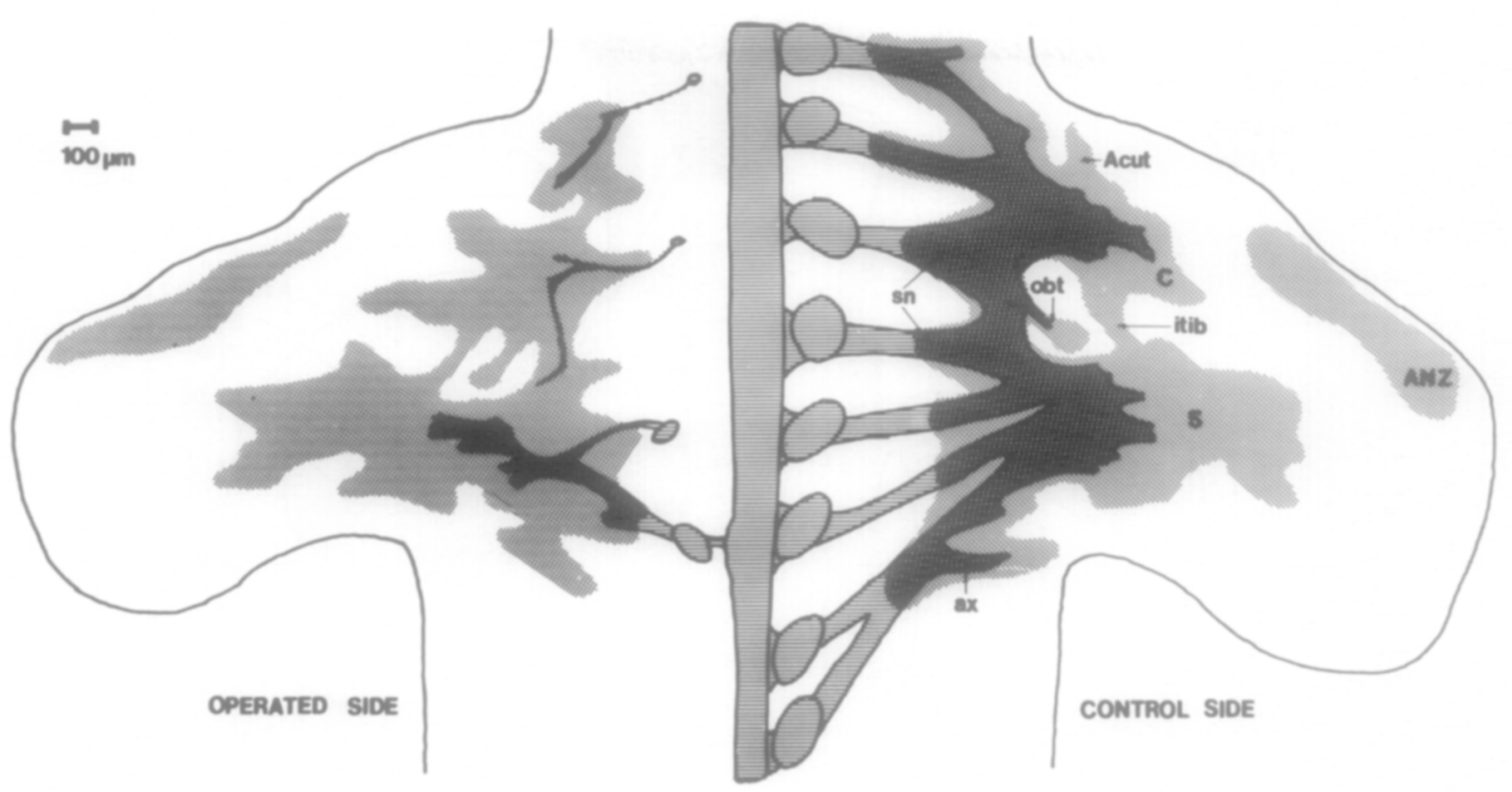

FIG. 5. Reconstruction of a representative operated embryo from a dorsal view. Neurite outgrowth was reduced to less than one-third of normal in the sciatic region (bottom left, also shown in Fig. 1B) and to less than one-tenth of normal in the crural region (top left; also shown in Fig. 1A). In fact, neurites could be detected in the crural region only with EM and their approximate distribution, as shown here, was obtained by sampling with EM. Neural tissuc is indicated by horizontal lines, the presence of phagocytes is indicated by stippling. On the control side (right) the lateral motor column, DRG, and patterns of nerve outgrowth are normal. Phagocytes are distributed along the spinal nerves (SN) in the anterior of each segment, within the plexus regions where the spinal nerves meet at the base of the limb, and extend beyond the tip of the outgrowing nerves into the pathways for the crural nerve trunk (C), sciatic nerve trunk (S), and axial (ax) and anterior cutaneous (Acut) nerves. Phagocytes were seen in the midlimb region between nerve trunk pathways only along the ventral obturator nerve trunk pathway (obt) and along the dorsal iliotibialis muscle nerve pathway (itib). Despite the extensive reduction of axonal outgrowth on the operated side, the spatial distribution of phagocytes is remarkably similar. ANZ, anterior necrotic zone.

where vascular endothelium is regressing. In the present preparations we could not determine whether vessels had regressed from the axon pathways or whether the cell remnants within phagocytes were endothelial.

Innervation of experimental limbs. We analyzed 12 sets of pathways (the crural and sciatic regions on six operated sides). In all cases, we conservatively estimated that innervation had been reduced to less than one-third of normal on the experimental side. Neurites were not detectable at the light microscopic level in three of these pathway regions. When these were examined ultrastructurally, a few neurites were found in the proximal plexus region (compare Figs. 7A and 7B) and we conservatively classified these as reduced to less than one-tenth of normal.

The source (sensory or motor) of the nerves that were present also varied. In six pathway regions, the lateral motor column was clearly deleted throughout the relevant segments and no axons were found in the ventral root region with EM. The nerve component in these consisted entirely of sensory neurites extending from one or two very small DRG (e.g., in the crural region shown in Figs. 5 and $6 \mathrm{~A}$ ). In the other six, a small portion of the motor column and a very reduced ventral root were found in one or two of the relevant segments (e.g., sciatic region in Fig. 5). One of these had no detectable sensory neurites and was considered to be totally motor. The other five had a small sensory component and were considered to be mixed nerves. It is not surprising that the sensory component was more often represented, since neural crest begins to migrate about the time our operations were done and it could also have been recruited from segments anterior or posterior to the deletion sites.

Distribution of phagocytes in experimental limbs. The distribution of phagocytes in experimental limbs was remarkably similar to that seen on the control sides, as illustrated in Figs. 5 and 6. In particular, phagocytes were distributed to a similar distal position along nerve trunk pathways, despite the fact that few neurites had entered the limb and none of these had colonized the limb to their normal distal extent. In the posterior portion of the crural region, phagocytes in the plexus region were again confined to a very small area and were not found dorsal or ventral to the ramus communicans pathway. Phagocytes were prominent within the spinal nerve pathway in the anterior sclerotome, within both plexus regions and their corresponding nerve trunk paths, along the cutaneous paths, and at foci within the muscle masses corresponding to muscle nerve pathways. Phagocytes were clearly absent in adjacent, non- 


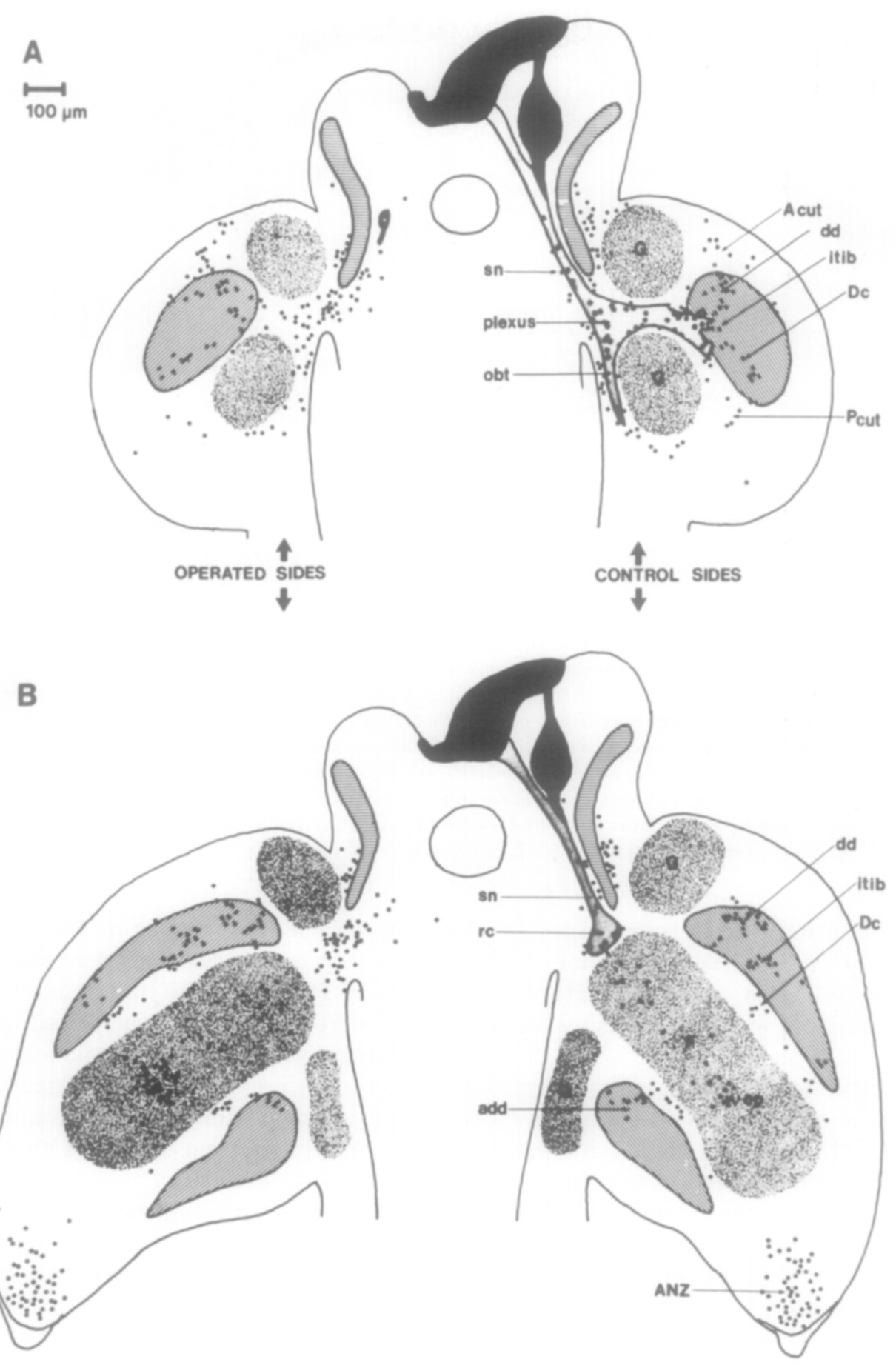

FIG. 6. Camera lucida tracings of (A) crural, (B) midlimb, (C) sciatic, and (D) posterior limb regions. The spinal cord and DRG (black), pattern and maximum extent of nerve outgrowth (stippling), muscle masses (diagonal lines), myotome (horizontal lines) pelvic girdle (G), and femur-tibiotarsus condensation $(F)$ were drawn from a representative section in each region. Sections were aligned with regard to the dorsal and ventral base of the limb and the positions of individual phagocytes in every other section through the region were indicated by black dots. In each drawing the experimental and control sides represent the same distance $(250-450 \mu \mathrm{m})$ along the anterior-posterior axis. These spatial patterns are discussed in the text. SN, spinal nerve. Nerve trunk pathways: obt, obturator (the ventral crural nerve trunk); Dc, dorsal crural; Ds, dorsal sciatic; Vs, ventral sciatic; ax, axial; rc, ramus communicans. Cutaneous pathways: Acut, anterior; Pcut, posterior. Muscle nerve pathways: dd, deep dorsal; itib, iliotibialis; add, adductors; ifib, iliofibularis; dv, deep ventral; ischio, ischioflexorius; caud, caudilioflexorius. Nonpathway regions: $A N Z$, anterior necrotic zone; OP, opaque patch. 


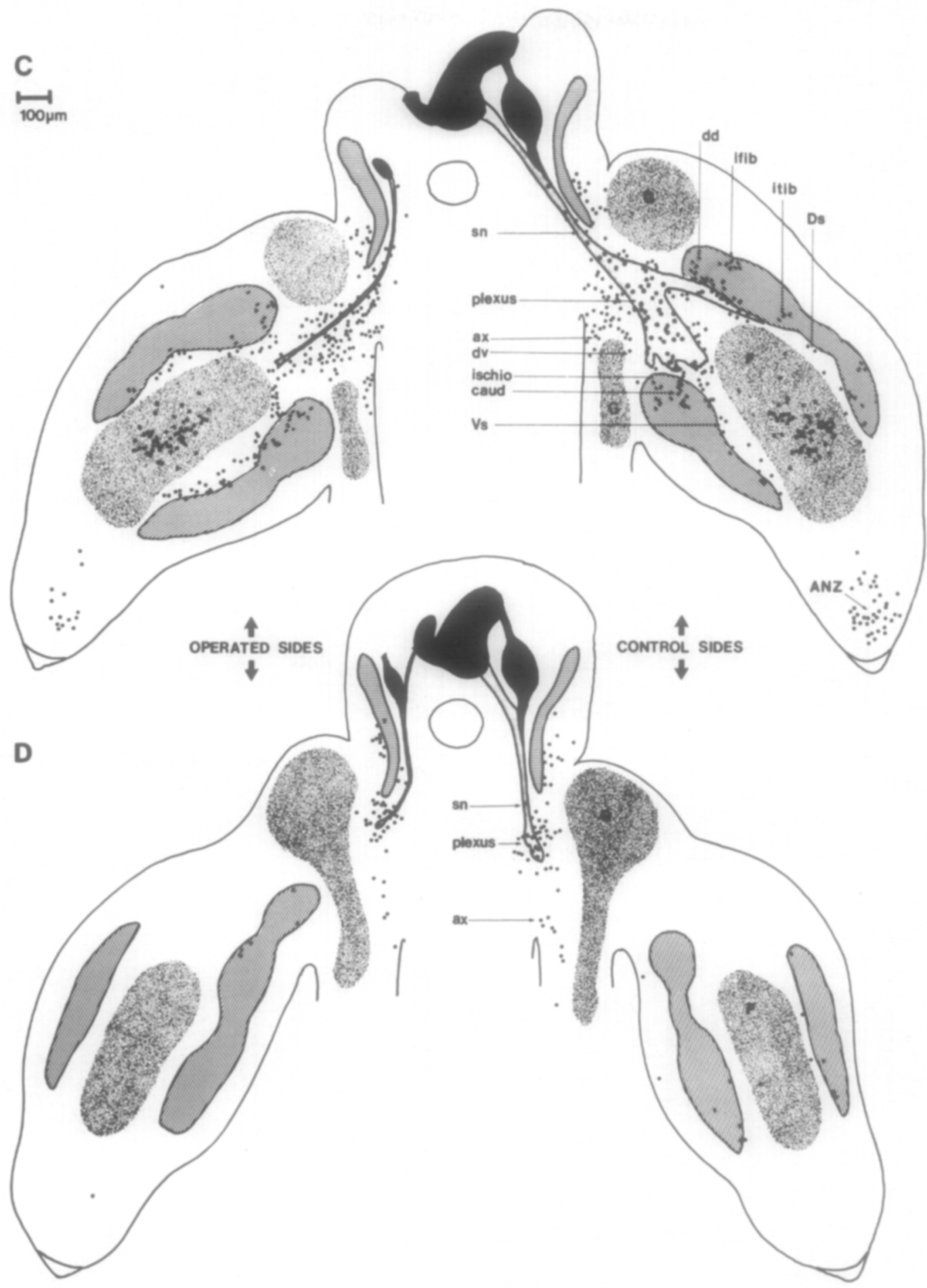

FIG. 6-Continued.

pathway regions. The pattern was similar in all respects to that seen in control limbs. The localization of phagocytes in the cartilage anlagen, the anterior necrotic zone, and lateral to the myotome was also unaffected by the operation. We conclude that the spatial distribution of phagocytes within the limb and, in par- ticular, along neurite pathways is independent of neurite outgrowth.

Abundance of phagocytes. We determined if there were any subtle dependence between the death of cells and neurite outgrowth by comparing the number of phagocytes in experimental and control limbs. We 

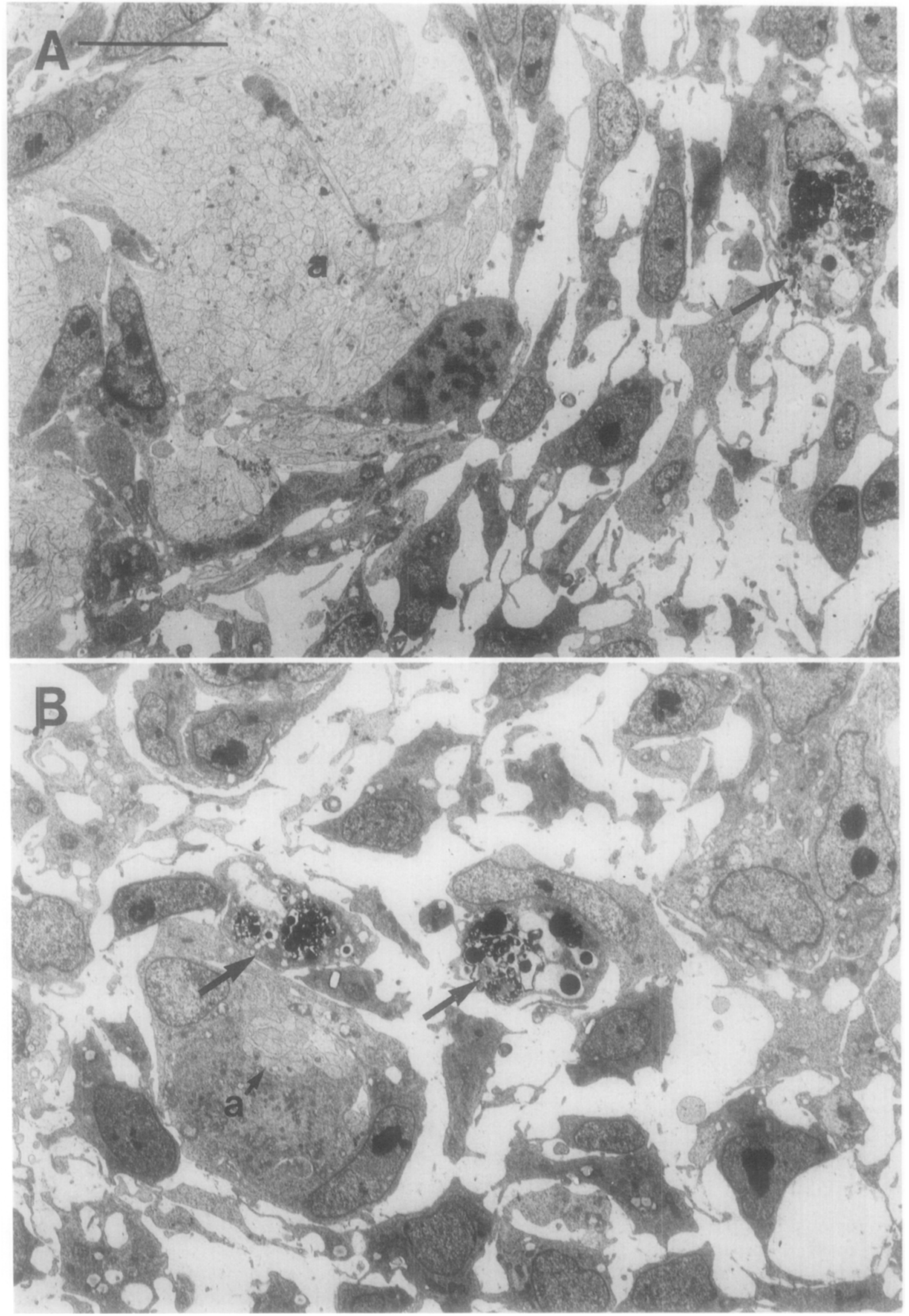

Fig. 7. Electron micrographs from the crural plexus regions of control (A) and operated (B) sides (see arrowheads in Fig. 1A). Large fascicles of axons (a) are visible on the control side. In contrast, very few axons were detected on the operated side. Phagocytes (arrows) were common on both sides. Calibration bar $=10 \mu \mathrm{m}$. 

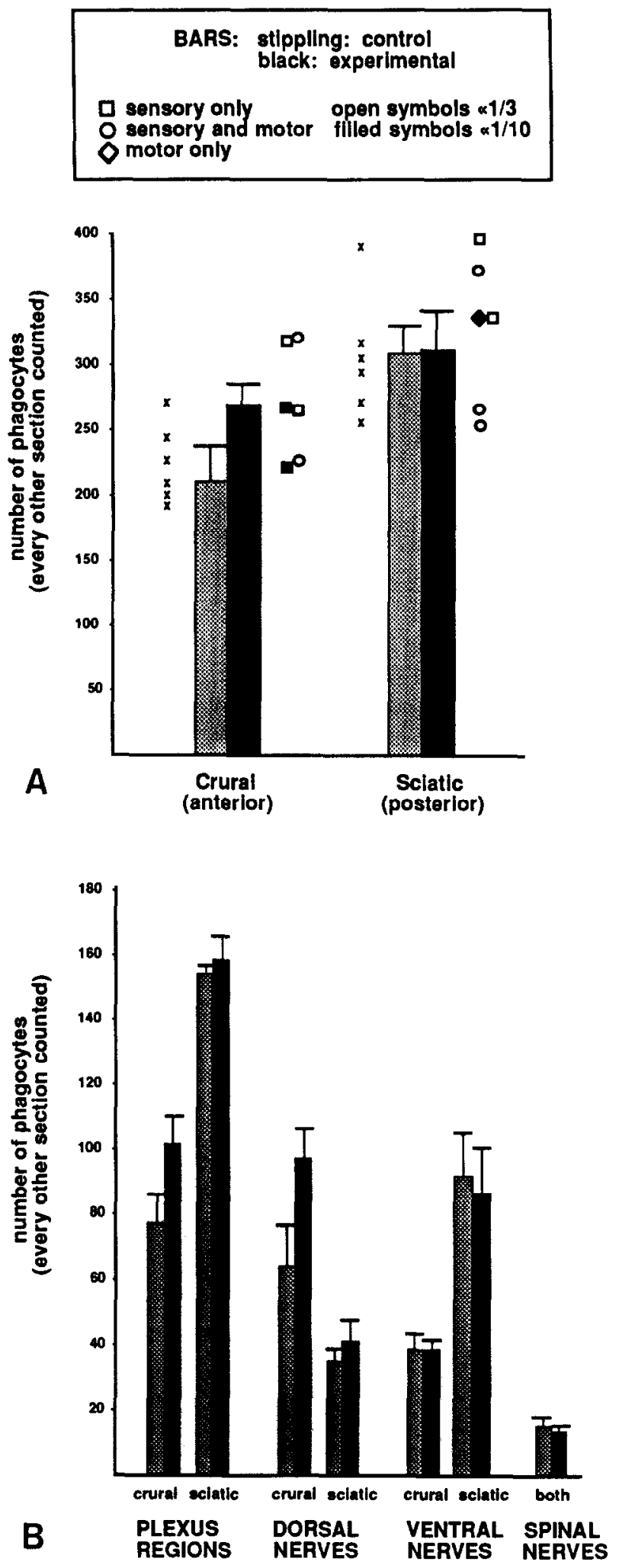

FIG. 8. The numbers of phagocytes along nerve pathways in control (stippling) and operated (black) limbs were determined from camera lucida tracings of every other section. (A) No significant difference was found in the numbers of phagocytes on control and experimental sides in either the crural or the sciatic regions. Symbols indicating individual experimental cases are coded in the key for the identity of the neurites and the extent of the depletion. Individual control values found no significant difference from the control in the numbers of phagocytes in either crural or sciatic regions, as shown in Fig. 8A. This figure also shows the values of individual cases for both control and experimental limbs. The latter are coded to show the extent of nerve depletion and whether the remaining neurites were sensory only, motor only, or mixed. There is clearly no trend for those cases with the most reduced innervation to have fewer phagocytes. In addition, the source of the innervation does not seem to matter.

We also determined the number of phagocytes within individual pathways in each region to examine the possibility that fewer phagocytes would be found in the more distal pathways that were generally uncolonized by neurites on the experimental side. This turned out not to be the case: there was no significant difference in numbers of phagocytes in comparable pathways (Fig. 8B). Again, the individual cases in which innervation was most reduced did not contain fewer phagocytes (not shown). In fact, there was a slight but nonsignificant trend for more phagocytes to be found in many of the paths on the experimental sides.

\section{DISCUSSION}

Death and axom guidance. We report here a remarkable correspondence between the spatial distributions of axon pathways and phagocytes at the stage when growth cones first enter the avian hindlimb. Phagocytes characterize both sensory and mixed neurite pathways; they are scattered like bread crumbs along a trail that extends far beyond the filipodial grasp of the most advanced growth cones; and they are largely absent in adjacent regions that growth cones do not enter. This correlation is an exciting one, since it is the first instance of an element that delineates axonal pathways within the limb. Although two molecules, laminin (Rogers et al., 1986) and NCAM (Tosney et al., 1986), appear in the environment before axon outgrowth and may generally enhance the suitability of the substratum for axon advance, neither of these precisely defines the axonal pathways. In addition, no other identifiable population and no particular arrangement of cells or extracellular matrix typifies the paths (Tosney and Landmesser, 1985a; Tosney, 1988b).

If the cell death we have documented is essential to growth cone guidance, it is likely to provide general cues

are indicated by $\times$. (B) The numbers of phagocytes on control and experimental sides did not significantly differ when each type of pathway (plexus region, dorsal path, ventral path, and spinal nerves) within crural and sciatic regions was compared. Dorsal and ventral pathways include the respective nerve trunk pathway with its associated cutaneous and muscle nerve pathways. 
that guide several populations of axons and is less likely to provide specific cues for the guidance of particular populations. For instance, the dying cells are scattered all along the pathways and are not concentrated in "decision regions" where motoneuron growth cones exhibit population-specific behaviors that are important to the precision of their projection (Tosney and Landmesser, $1985 \mathrm{~b}, \mathrm{c})$. In addition, phagocytes are present along sensory as well as mixed paths and so are likely to be relevant to the guidance of both populations. Phagocytes are also seen within anterior sclerotome. While this environment is important to the overall pattern of axonal outgrowth, it provides no essential cues for specific motor innervation (Lance-Jones, 1988; Tosney, 1987, 1988a).

Necrosis of cell populations has been proposed in other systems to correlate with the formation of axonal pathways. For instance, a wave of cell death in the invaginating otic cup is associated with release of cells from the epithelium and the subsequent formation of a "funnel" of aggregated cells along which auditory axons grow (Carney and Silver, 1983). Within the retina, a wave of cell necrosis may enlarge intercellular spaces that may guide the outgrowth of retinal ganglion axons; in embryos with inherited optic nerve aplasia, death is reduced, space is diminished, and axons do not grow out (Silver and Robb; 1979). When superficial layers of one side of the superior colliculus are removed, the optic axons that normally innervate this contralateral target are reported to travel across a "necrotic tissue bridge" to innervate target on the ipsilateral side (So, 1979). This induced cell death may provide a novel pathway or remove a barrier to outgrowth. In contrast, Silver et al. (1987) demonstrate a case in which cell death is associated with the formation of a barrier. A tightly localized zone of necrosis presages a local increase in the density of cells; this condensation may act as an axon boundary that separates the optic and olfactory axons.

How could cell death play an essential and direct role in axonal pathfinding within the limb? The death of cells might alter the intercellular density of tissues along pathways, leading to increased space as it apparently does in the retina. While there is no obvious difference in the intercellular spacing on pathways and in adjacent regions (cf. Tosney and Landmesser, 1985a), differences in cell density could be transient or subtle and require morphometric quantitation to detect. Death of cells could also play a direct role if growth cones were attracted to necrotic debris or to the accumulation of recycled by-products (see also Silver, 1976) or if released substances altered the local environment and enhanced its suitability as a substratum. Although these mechanisms are plausible, it remains to be seen whether the death of cells in any system is actually essential to axon outgrowth. If it is, it should be possible to entice growth cones into novel regions by killing cells or to hinder axon outgrowth by preventing cell death. We have begun these types of experiments.

The relationship between cell death and axon outgrowth is as likely to be indirect. Permissive regions evidently emerge in a limb that is initially a hostile environment for growth cone advance (Tosney and Landmesser, 1985a; Landmesser, 1987). The phagocytosis we see may merely mark the emergence of pathways that have become suitable for axonal outgrowth for reasons other than the presence of dying or phagocytized cells.

The paths not traveled by. Several sites of necrosis within the limb do not correspond to axonal pathways. This section discusses reasons for believing these sites to be inaccessible to axons. The first of these sites, the anterior necrotic zone, is distant to and discontinuous with axonal pathways. If cell death aids growth cone advance by altering the local environment, this site of death is too distant to be relevant. If necrosis is chemotactic for growth cones, this site could generally promote distal growth.

Phagocytosis is prominent within cartilage primordia, particularly in the "opaque patch" between the femur and tibiotarsus primordia. Despite the death of these cells, growth cones do not invade the central cartilagenous core of the limb. These regions of cell death may not provide pathways because chondrocytes, rather than some other type of cell, are dying and these cells do not present the correct signals. Alternatively, the precartilagenous environment may prevent growth cone advance and negate any response to the death of cells deep within the primordium. In fact, precartilagenous tissue in general has been hypothesized to act as a barrier to axonal outgrowth (cf. Tosney, 1988a) and there is experimental evidence that three such tissues (the pelvic girdle precursor, the posterior sclerotome, and the ventromedial sclerotome within both the anterior and posterior of a segment) do inhibit axon advance (Tosney and Landmesser, 1984; Keynes and Stern, 1984, Tosney, 1988a).

Phagocytosis is also seen within developing dermis near the ventrolateral myotome. Growth cones in the adjacent plexus region would have to turn at very acute angles to enter this region, and this may hinder their colonization of this area. It is probably more germaine that this region of cell death shares features with precartilagenous tissues. Two substances that bind to precartilagenous barrier tissues, alcian blue and peanut agglutinin lectin, also bind to the developing dermal mesenchyme (Tosney and Landmesser, 1985a; Oakley and Tosney, 1988; Oakley and Tosney, unpublished observations). Molecules typical of cartilagenous tissues 
are also expressed at the dorsal midline of the spinal cord in a region that acts as a barrier to axon advance (Jerry Silver, personal communication). The dermis may, therefore, also act as a barrier to axon outgrowth due to its expression of substances that are more commonly thought to be associated with cartilage differentiation but that act to inhibit axon advance.

What kills cells along axonal pathways? We have also shown that both the position and number of phagocytes in the limb are independent of growth cone outgrowth. Despite the fact that a few neurites had entered the plexus region in our most extensive deletions, it is clear that a contact-mediated interaction between growth cones and cells is not essential to the patterned death and phagocytosis of cells along the pathways. Any more distant interaction also seems unlikely to be essential. If growth cones released a "killer" substance, we would have to hypothesize that only a few growth cones were required to catalyze an extraordinarily complex and far reaching pattern of cell death and, moreover, were able to kill cells in their normal numbers. It is difficult to see how this could be accomplished.

It is far more likely that the patterns of cell death that characterize the axonal pathways are a function of limb-dependent and nerve-independent processes. Cell death may be occasioned by developmental processes that are primarily related to the development of limb tissues. Below we briefly discuss and evaluate the possible role of three morphogenetic processes commonly associated with localized cell death: the sculpting and separation of tissues, histogenesis, and the regression of developmental structures. (These processes are reviewed by Glucksmann, 1951; Saunders, 1966; Oppenheim, 1985.)

One plausible possibility is that cells are locally phagocytized at sites where limb tissues separate from one another. Phagocytosis is seen at the boundaries between several tissues that are first becoming distinct as axons enter the limb; some of these planes correspond to axonal pathways and some do not. While the presence of phagocytosis within anterior but not posterior sclerotome does not seem, at first glance, to be an example of tissue delineation, it is also explicable in these terms if the dorsal and ventral portions of anterior sclerotome are distinct populations, and there is some evidence that this is the case (see Tosney, 1988a). The fact that many axonal pathways do lie within emerging tissue planes suggests that at least a portion of the cell death that characterizes axonal pathways could be primarily associated with the initial definition of tissues. However, at the stage we examined, phagocytes were often present only along the portion of a particular intertissue plane that corresponded to a neurite pathway. It is possible that phagocytosis was more widespread earlier and that the spatial pattern of cell death at this stage is a result of a pattern of tissue separation that is highly stereotyped in space and in time. We are characterizing the developmental time course of phagocytosis in these regions to determine if this is the case.

The foci of phagocytosis within the muscle masses that correspond to sites where muscle nerves will form are not explicable in terms of tissue delineation. While the muscle masses will separate and form individual muscles a day or two after they are innervated, and myofilament-containing cells are selectively phagocytized between the emerging muscles (Schroeter and Tosney, 1987), the foci of phagocytosis we observed in the current study do not correspond spatially to the planes where muscles will separate. Death in these foci may be an example of the histogenetic cell death that is commonly supposed to accompany the differentiation of many tissues. Muscle cells are required for muscle nerves to form (Lewis et al., 1981) and it may be the programmed death of some of these cells that we see. If it does turn out that two different developmental processes (at muscle nerve foci and between emerging tissues) contribute to patterned cell death, then the most parsimonious explanation of the coincidence between cell death and pathways is that cell death itself is directly important to the guidance of growth cones.

Another sort of remodeling could explain the localization of phagocytes both at emerging tissue borders and within foci in the muscle mass. Cells that are phagocytized along axonal pathways could be the remnants of regressing blood vessels. The vasculature pattern is known to change as the forelimb tissues develop (reviewed by Caplan, 1985). However, little is known of the precise distribution of the early vasculature or of the local alterations in vasculature patterns that accompany the maturation of the hindlimb tissues. The possibility that growth cones follow a trail that is demarked by the previous colonization and regression of blood vessels is an intriguing one that we are currently investigating.

It is important to point out that cell death, even if it is a result of some other process, could still play an essential role in axon guidance. Growth cones are thought to respond to features in their environment that emerge as a side effect of developmental processes and that are not laid down only or primarily for axonal guidance (Palka, 1987; Tosney, 1988a). Indeed, despite the close spatial correspondence, it seems rather unlikely that such a complex pattern of cell death has been programmed into the embryo solely for the benefit of outgrowing neurites. Growth cones may for some reason require the death of cells to advance into a region, but the death itself may be a consequence of or mediate other developmental events. Determining what devel- 
opmental processes are associated with this patterned cell death, identifying the population that dies, charting the spatial-temporal distribution of cell death, and, in particular, experimentally altering the pattern of cell death should help us to elucidate its role during axonal pathfinding in this system.

This work was supported by National Institute of Health Grant NS-21308. We thank Sophia Tyrrell for technical assistance, Robert Oakley for reading the manuscript, and David Bay for photographic assistance.

\section{REFERENCES}

CAPLAN, A. I. (1985). The vasculature and limb development. Cell Differ. 16, 1-11.

CARney, P. R., and Silver, J. (1983). Studies on cell migration and axon guidance in the developing distal auditory system of the mouse. J. Comp. Neurol. 215, 359-369.

DAWD, D. S., and HiNCHLIFFE, J. R. (1971). Cell death in the "opaque patch" in the central mesenchyme of the developing chick limb: A cytological, cytochemical and electron microscopic analysis. $J . \mathrm{Em}$ bryol. Exp. Morphol. 26, 401-424.

GluCKSManN, A. (1951). Cell deaths in normal vertebrate ontogeny. Biol. Rev. Cambridge Philos. Soc. 26, 59-86.

Hamburger, V., and Hamilton, H. L. (1951). A series of normal stages in the development of the chick embryo. J. Morphol. 88, 49-82.

JACOB, M., CHRIST, B., and JACOB, H. J. (1979). The migration of myogenic cells from the somites into the leg region of avian embryos. An ultrastructural study. Anat. Embryol. 157, 291-309.

KEYNES, R. J., and STERN, C. D. (1984). Segmentation in the vertebrate nervous system. Nature (London) 310, 786-789.

KRYostoseK, A., and SEEDS, N. W. (1981). Plasminogen activator release at the neuronal growth cone. Science 213, 1532-1534.

LANCE-JONES, C. J. (1988). The effect of somite manipulation on the development of motoneuron projection patterns in the embryonic chick hindlimb. Dev. Biol. 126, 408-419.

LANDMESSER, L. T. (1984). The development of specific motor pathways in the chick embryo. Trends Neurosci. 7, 336-339.

LANDMESSER, L. T. (1987). Peripheral guidance cues and the formation of specific motor projections in the chick. In "From Message to Mind: Directions in Developmental Neurobiology" (S. S. Easter, K. F. Barald, and B. M. Carlson, Eds.), pp. 121-133. Sinauer, Sunderland, MA.

LANDMESSER, L. T., and HoNIG, M. (1986). Altered sensory projections in the chick hind limb following the early removal of motoneurons. Dev. Biol. 118, 511-531.

Lewis, J., Chevallier, A., KienY, M., and Wolpert, L. (1981). Muscle nerve branches do not develop in chick wings devoid of muscle. $J$. Embryol. Exp. Morphol. 64, 211-232.

OAKLEY, R., and TOSNEY, K. W. (1988). Peanut agglutinin (PNA) binds to tissues that act as barriers to axon advance in the chick embryo. Soc. Neurosci. Abstr. 14, 870.

OPPENHEIM, R. W. (1985). Naturally occurring cell death during neural development. Trends Neurosci. 8, 487-493.

PALKA, J. (1987). Axon guidance in the insect periphery. Development 99, 307-309.

PATTERSON, P. H. (1985). On the role of proteases, their inhibitors and the extracellular matrix in promoting neurite outgrowth. J. Phy siol. (Paris) 80, 207-211.

PitTman, R. N. (1985). Release of plasminogen activator and a calcium-dependent matalloprotease from cultured sympathetic and sensory neurons. Dev. Biol. 110, 91-101.
Rogers, S. L., Edson, K. J., Letourneau, P. C., and Mcloon, S. C. (1986). Distribution of laminin in the developing peripheral nervous system of the chick. Dev. Biol 113, 429-435.

SAUNDERS, J. W., JR. (1966). Death in embryonic systems. Science 154, 604-612.

Saunders, J. W., JR., and Fallon, J. F. (1967). Cell death in morphogenesis. In "Major Problems in Developmental Biology." (M. Locke, Ed.), pp. 289-314. Academic Press, New York.

Saunders, J. W., JR., Gasseling, M. T., and SAunders, L. C. (1962). Cellular death in morphogenesis of the avian wing. Dev. Biol. $\mathbf{5}$, 147-178.

Schroeter, S., Pokrzywinski, J. A., and Tosney, K. W. (1988). Mesenchymal cell death delineates axon pathways in the hindlimb and does so independently of neural interactions. Soc. Neurosci. Abstr. 14,870 .

SCHROETER, S., and TosNEy, K. W. (1987). Formation of individual muscles in the chick thigh. Soc. Neurosci. Abstr. 13, 253.

SILVER, J. (1976). A study of ocular morphogenesis in the rat using $\left[{ }^{3} \mathrm{H}\right]$ thymidine autoradiography: Evidence for thymidine recycling in the developing retina. Dev. Biol. 49, 487-495.

Silver, J., Poston, M., and Rutishauser, U. (1987). Axon pathway boundaries in the developing brain. I. Cellular and molecular determinants that separate optic and olfactory projections. $J$. Neurosci. 7, 2264-2272.

SILVER, J., and RoBB, R. M. (1979). Studies on the development of the eye cup and optic nerve in normal mice and in mutants with congenital optic nerve aplasia. Dev. Biol. 68, 175-190.

So, K.-F. (1979). Development of abnormal recrossing retinotectal projections after superior coliculus lesions in newborn Syrian hamsters. J. Comp. Neurol. 186, 241-258.

STIRLING, R. V., and Summerbell, D. (1985). The behavior of growing axons invading developing chick wing buds with dorsoventral or anteroposterior axis reversed. J. Embryol. Exp. Morphol. 85, 251-269.

Swanson, G. J., and Lewis, J. (1982). The timetable of innervation and its control in the chick wing bud. J. Embryol. Exp. Morphol. 71, 121-137.

Tosney, K. W. (1987). Proximal tissues and patterned neurite outgrowth at the lumbosacral level of the chick embryo: Deletion of the dermamyotome. Dev. Biol. 122, 540-558.

Tosney, K. W. (1988a). Proximal tissues and patterned neurite outgrowth at the lumbosacral level of the chick embryo: Partial and complete deletion of the somite. Dev. Biol. 127, 266-286.

TOSNEY, K. W. (1988b). The somites and axon guidance. Scanning Microsc. 2, 427-442.

Tosney, K. W., and Landmesser, L. T. (1984). Pattern and specificity of axonal outgrowth following varying degrees of chick limb bud ablation. $J$. Neurosci. 4, 2158-2527.

Tosney, K. W., and LANDmesser, L. T. (1985a). Development of the major pathways for neurite outgrowth in the chick hindlimb. Dev. Biol. 109, 193-214.

TOSNEY, K. W., and LANDMESSER, L. T. (1985b). Growth cone morphology and trajectory in the lumbosacral region of the chick embryo. $J$. Neurosci. 5, 2345-2358.

TOSNEY, K. W., and LANDMESSER, L. T. (1985c). Specificity of motoneuron growth cone outgrowth in the chick embryo. J. Neurosci. 5, 2336-2344.

TOSNEY, K. W., and LANDMESSER, L. T. (1986). Neurites and growth cones in the chick embryo: enhanced tissue preservation and visualization of HRP-labeled subpopulations in serial $25-\mu \mathrm{m}$ plastic sections cut on a rotary microtome. $J$. Hislochem. Cytochem. 34, 953-957.

TOSNEY, K. W., Watanabe, M., Landmesser, L., and RUTiShaUSer, U. (1986). The distribution of NCAM in the chick hindlimb during axon outgrowth and synaptogenesis. Dev. Biol. 114, 437-452. 